\title{
Efeitos do treinamento físico na pressão arterial de adolescentes com sobrepeso e obesidade: Uma revisão sistemática
}

\author{
Effects of exercise training on blood pressure in overweight and obese adolescents: A systematic \\ review
}

Efectos de entrenamiento físico en la presión arterial de adolescentes con sobrepeso y obesidad: Una revisión sistemática

\section{Resumo}

O objetivo desta revisão foi analisar os efeitos do treinamento físico na pressão arterial de adolescentes com sobrepeso e obesidade, buscando identificar a magnitude do efeito em relação ao tipo de treinamento. A presente revisão utilizou as bases de dados do PUBMED, SCOPUS, WEB OF SCIENCE, COCHRANE LIBRARY e LILACS. Os critérios de inclusão foram: (1) Ensaios Clínicos Randomizados; (2) Adolescentes com sobrepeso e obesidade; (3) Mínimo de 8 semanas de Intervenção com treinamento físico; (4) medidas de resultados de pressão arterial pré e pós treinamento; (5) grupo controle sem intervenção e mantendo seus hábitos diários. Os estudos selecionados foram analisados em sua qualidade metodológica pela escala PEDro. Oito artigos, de um total de 625, atenderam aos critérios de inclusão, totalizando 249 participantes. Três estudos se utilizaram do treinamento físico aeróbio como intervenção, dois do treinamento físico de força e quatro estudos da combinação dos treinamentos, aeróbio e de força, para responderem seus objetivos. Dos trabalhos selecionados, cinco apresentaram reduções significativas em seus valores de pressão arterial após intervenção do treinamento, principalmente na PAS. Os resultados sugerem que o treinamento físico é capaz de reduzir os valores de pressão arterial, independentemente do tipo, sendo ele de características aeróbias, de força, ou a combinação das duas. Embora os valores de pressão arterial tendam a apresentar maior redução quando realizado o treinamento aeróbio em comparação ao treinamento de força ou combinado, a literatura carece de consenso sobre as características de cada tipo de treinamento e a melhor estratégia de prescrição.

Palavras-chave: Adolescentes; Obesidade; Pressão arterial; Treinamento físico.

\section{Abstract}

The aim of this review was to analyze the effects of physical training on the blood pressure of overweight and obese adolescents, searching to identify the magnitude of the effect in relation to the type of training. The present review 
used PUBMED, SCOPUS, WEB OF SCIENCE, COCHRANE LIBRARY and LILACS as databases. Inclusion criteria were: (1) Randomized Clinical Trials; (2) Overweight and obese adolescents; (3) Minimum 8 weeks of intervention with physical training; (4) pre and post-training blood pressure outcome measures; (5) control group without intervention and keeping their daily habits. The selected studies were analyzed for their methodological quality using the PEDro scale. Eight articles, out of a total of 625, met the inclusion criteria, totaling 249 participants. Three studies used aerobic physical training as an intervention, two used strength training and four studies used a combination of aerobic and strength training to meet their goals. Of the selected works, five showed significant reductions in their blood pressure values after training intervention, especially in SBP. The results suggest that physical training is capable of reducing blood pressure values, regardless of the type, whether it has aerobic characteristics, strength, or a combination of the two. Although blood pressure values tend to show a greater reduction when aerobic training is performed compared to strength training or combined training, the literature lacks consensus on the characteristics of each type of training and the best prescription strategy.

Keywords: Adolescents; Obesity; Blood pressure; Physical training.

\section{Resumen}

El objetivo de esta revisión fue analizar los efectos de entrenamiento físico sobre la presión arterial de adolescentes con sobrepeso y obesidad, buscando identificar la magnitud del efecto en relación al tipo de entrenamiento. Esta revisión utilizó las bases de datos PUBMED, SCOPUS, WEB OF SCIENCE, COCHRANE LIBRARY y LILACS. Los criterios de inclusión fueron: (1) Ensayos clínicos aleatorizados; (2) Adolescentes con sobrepeso y obesidad; (3) Mínimo 8 semanas de intervención con entrenamiento físico; (4) medidas de resultado de la presión arterial antes y después de entrenamiento; (5) grupo control sin intervención y manteniendo sus hábitos diarios. Los estudios seleccionados fueron analizados por su calidad metodológica mediante la escala PEDro. Ocho artículos, de un total de 625, cumplieron los criterios de inclusión, totalizando 249 participantes. Tres estudios utilizaron entrenamiento físico aeróbico como intervención, dos utilizaron entrenamiento de fuerza y cuatro estudios utilizaron una combinación de entrenamiento aeróbico y de fuerza para alcanzar sus objetivos. De los trabajos seleccionados, cinco mostraron reducciones significativas en sus valores de presión arterial después de la intervención de entrenamiento, especialmente en la PAS. Los resultados sugieren que el entrenamiento físico es capaz de reducir los valores de presión arterial, independientemente del tipo, ya sea que tenga características aeróbicas, fuerza o una combinación de ambas. Si bien los valores de presión arterial tienden a mostrar una mayor reducción cuando se realiza entrenamiento aeróbico en comparación con el entrenamiento de fuerza o el entrenamiento combinado, la literatura carece de consenso sobre las características de cada tipo de entrenamiento y la mejor estrategia de prescripción.

Palabras clave: Adolescentes; Obesidad; Presión arterial; Entrenamiento físico.

\section{Introdução}

A obesidade é um grande problema de saúde pública, com mais de um bilhão de pessoas com sobrepeso ou obesas em todo o mundo, segundo a Organização Mundial da Saúde (OMS) (WHO, 2020). Uma doença crônica complexa com proporções epidêmicas resultante de fatores biogenéticos, comportamentais, ambientais e econômicos que representa um fenômeno social real e cujo manejo muitas vezes é difícil e requer multidisciplinaridade (García-Hermoso et al., 2015;MilanoGai et al., 2018). Estudos epidemiológicos têm mostrado um rápido aumento na prevalência de sobrepeso e obesidade, globalmente, o número de casos de obesidade quase triplicou desde 1975, não apenas em adultos, mas também em crianças e adolescentes, aumentando assim o risco de desenvolver doença cardiovascular precoce e comorbidades (Vasconcellos et al., 2014; WHO, 2020). De acordo com a OMS, o número de crianças e adolescentes com obesidade aumentou de 11 milhões para 124 milhões nas últimas quatro décadas. Para o Centers for Disease Control and Prevention (CDC), a prevalência de obesidade mais do que dobrou em crianças e quadruplicou em adolescentes nas últimas três décadas (Bharath et al., 2018; Cvetković et al., 2018; WHO, 2018).

A obesidade em crianças e adolescentes tem consequências deletérias, tanto em curto quanto em longo prazo. Riscos de saúde mais imediatos da obesidade infantil estão associados a um risco aumentado de pré-diabetes, problemas musculoesqueléticos e anormalidades pulmonares (por exemplo, apneia obstrutiva do sono e asma) e psicossociais (por exemplo, baixa autoestima, ansiedade, depressão e disfunção social). Há também um alto risco de que um adolescente obeso tenha $80 \%$ de chance de se tornar um adulto obeso (Sigal et al., 2014; Monteiro et al., 2015; García-Hermoso et al., 2020). A persistida elevação das concentrações de adipocinas inflamatórias, oriundas do excesso de adiposidade, tem sido associada a 
fatores aumentados de riscos de diversos desfechos crônicos na saúde de crianças e adolescentes, como resistência à insulina, diabetes tipo 2, dislipidemias, disfunção endotelial, processo aterosclerótico prematuro, esteatose hepática não alcoólica, disfunção precoce do sistema nervoso autônomo, rigidez arterial, hipertensão e síndrome metabólica (Vasconcellos et al., 2014; Antunes et al., 2015; Dias et al., 2015; Kelly et al., 2015; Farinatti et al., 2016; Bharath et al., 2018; Lee et al., 2019).

Como é de se notar, a hipertensão, principal fator de risco para doenças cardiovasculares, também é altamente prevalente em adolescentes, principalmente os obesos, chegando a prevalências seis vezes maiores em adolescentes obesos comparados aos seus pares com peso normal. De fato, estudos clínicos e epidemiológicos apontam para a influência da obesidade na pressão arterial mesmo na infância. Isso é particularmente relevante porque a hipertensão arterial em repouso em crianças e adolescentes pode acompanhar até a idade adulta (Farah et al., 2012; García-Hermoso et al., 2013).

Independentemente da idade, o exercício físico e o controle alimentar são amplamente prescritos para o tratamento da obesidade. Além disso, há uma grande variedade de protocolos de exercícios direcionados à perda de peso, embora sua eficácia não seja clara quando aplicados à população pediátrica (Antunes et al., 2015). Em crianças e adolescentes obesos, tanto o treinamento físico aeróbio (TA) quanto o treinamento físico de força (TF) reduzem os valores de pressão arterial e melhoram as concentrações de lipoproteína de baixa densidade e triglicerídeos, dilatação mediada por fluxo, insulina, glicose em jejum e composição corporal (García-Hermoso et al., 2020). O exercício aeróbio é um modelo comumente recomendado para indivíduos obesos, com efeitos benéficos na composição corporal, perfil lipídico, pressão arterial, controle glicêmico e aptidão cardiorrespiratória. Em comparação, o treinamento de força aumenta a própria força, massa e resistência musculares, e aumenta a densidade mineral óssea. Acredita-se que o treinamento combinado (TC) projetado para ativar as duas vias metabólicas diferentes (aeróbica e anaeróbica), aumenta os efeitos dos modelos de treinamento aeróbio e de força isolados (Monteiro et al., 2015). Embora os exercícios físicos pareçam desempenhar um papel claro na promoção da saúde cardiovascular, sua relação com a pressão arterial em crianças obesas é menos clara. Uma melhor compreensão do papel que desempenha nas adaptações cardiovasculares dessas crianças pode ajudar a projetar melhores estratégias para reduzir o risco cardiovascular nessa população.

Portanto, o objetivo desta revisão foi analisar os efeitos do treinamento físico na pressão arterial de adolescentes com sobrepeso e obesidade, buscando identificar a magnitude do efeito em relação ao tipo de treinamento, observando também o efeito dos métodos de treinamento e as características dos participantes.

\section{Metodologia}

Trata-se de uma revisão sistemática de ensaios clínicos randomizados controlados (ECR), de acordo com os padrões estabelecidos pelas Diretrizes Metodológicas - elaboração de revisão sistemática e metanálise de ensaios clínicos randomizados, elaboradas pelo Ministério da Saúde do Governo do Brasil (BRASIL, 2012) e pela declaração PRISMA Preferred Reporting Items for Systematic Reviews and Metanalyses (Liberati et al., 2009; Moher et al., 2009; Hutton et al., 2015). A pergunta da busca bibliográfica desta revisão foi: "Quais tipos de treinamento físico têm mais efetividade na redução dos níveis pressóricos em repouso de adolescentes com sobrepeso/obesidade?”. Dessa forma a estratégia PICO foi elaborada, e consequentemente definiram-se os critérios de elegibilidade dos estudos.

\section{Critérios de elegibilidade dos estudos}

Foram considerados elegíveis para o presente estudo somente ensaios clínicos randomizados e controlados, publicados nos idiomas inglês, português e espanhol, sem restrição para o período de publicação, realizados em adolescentes de 10-19 anos (WHO, 2017) com diagnóstico de sobrepeso/obesidade (IMC > percentil 85) (SBP, 2019) submetidos à no mínimo 08 semanas de treinamento físico- resistido, aeróbio ou a combinação destes (em que o grupo controle não recebeu 
nenhum tipo de orientação ou intervenção com exercícios físicos, nutrição, restrição alimentar ou tratamento medicamentoso), valores de pressão arterial em repouso antes e após período de intervenção relatado no estudo e com grupo controle mantendo seus hábitos diários de dieta e atividades físicas. Foram excluídos os estudos com ausência de grupo controle, outros que não informaram valores de pressão arterial, e aqueles que não foram localizados por inconsistência na sua referência, tornando o artigo indisponível nas bases de dados conhecidas.

\section{Fontes utilizadas e estratégia de busca}

Os artigos foram pesquisados, de agosto a outubro de 2020, nas bases de dados do PUBMED, SCOPUS, WEB OF SCIENCE, COCHRANE LIBRARY e LILACS por meio de descritores listados nos Descritores de Ciências em Saúde (DeCS) e no Medical Subject Headings (MeSH). A estratégia de busca foi composta pela população (adolescentes com sobrepeso/obesidade), intervenção/comparação (treinamento físico de força, treinamento físico aeróbio e a combinação dos dois) e o desfecho (efeito do treinamento nos valores de pressão arterial em repouso). A conexão entre os termos escolhidos para a estratégia de busca foi estabelecida pelos operadores booleanos AND, OR e NOT. A estratégia de busca utilizada na base de dados do PUBMED está representada pelo Quadro 1. Nas demais bases de dados, a utilização dos recursos booleanos, bem como a estratégia foi adaptada de acordo com a disponibilidade de busca a partir dos termos presentes no MeSH ou em seus correspondentes no DeCS, de forma a garantir a obtenção do maior número de ensaios clínicos possíveis.

Quadro 1. Estratégia de busca utilizada para a base de dados PUBMED (outubro 2020).

((((Adolescent[mh] OR Adolescent[tiab] OR Adolescence[tiab] OR Teen*[tiab] OR Youth*[tiab] OR Child[mh] OR Child*[tiab]) AND (Obesity[mh] OR Obesity[tiab] OR "pediatric obesity"[mh] OR "pediatric obesity"[tiab] OR Obesity, Abdominal[mh] OR Obesity, Abdominal[tiab] OR Overweight[mh] OR Overweight[tiab])) OR ("Adolescent Overweight"[tiab] OR "Overweight, Adolescent"[tiab] OR "Adolescent Obesity"[tiab] OR "Obesity, Adolescent"[tiab] OR "Obesity in Adolescence"[tiab])) AND (("Resistance Training"[mh] OR "Resistance Training"[tiab] OR "Strength Training"[tiab] OR "Weight training"[tiab] OR "resisted training"[tiab] OR "isometric training"[tiab]) OR ("Endurance Training"[mh] OR "Endurance Training"[tiab] OR "aerobic exercise"[tiab] OR "combined exercise"[tiab] OR Running[mh] OR Running[tiab] OR Swimming[mh] OR Swimming[tiab] OR Walking[mh] OR Walking[tiab]))) AND (("Blood Pressure"[mh] OR "Blood Pressure"[tiab] OR "Arterial Pressure"[mh] OR "Arterial Pressure"[tiab] OR "Post-exercise Hypotension"[mh] OR "Post-exercise Hypotension"[tiab] OR "Prehypertension"[mh] OR "Prehypertension"[tiab] OR hypertension[mh] OR hypertension[tiab]))

Fonte: Autores.

\section{Seleção dos estudos}

A seleção dos estudos respeitou três fases: 1) leitura dos títulos e resumos; 2) leitura do texto na íntegra; e 3) análise qualitativa dos estudos. Na primeira fase, foram selecionados os artigos com pelo menos dois dos termos principais da busca explícitos no título ou seus sinônimos, identificados no MeSH (para os estudos em inglês) ou em DeCS (para os estudos em português e espanhol), quando possível. Após seleção na primeira fase, os registros foram exportados para o programa gerenciador de referências Mendeley®, onde a duplicidade de artigos foi avaliada, sendo considerada só uma versão. Resumos foram avaliados na sequência a fim de confirmarem adequação temática. Esta etapa inicial foi realizada por dois revisores independentes, que classificaram os estudos como incluído, excluído ou duvidoso. Aqueles classificados como duvidosos 
foram revistos e discutidos por um terceiro revisor, para obtenção de um consenso. Assim, os artigos que atenderam aos critérios de elegibilidade seguiram para a fase seguinte.

$\mathrm{Na}$ segunda fase, relacionada à leitura do texto na íntegra, foi realizada a confirmação da adequação temática e o respeito aos critérios de elegibilidade. Os estudos que atenderam a tais critérios foram incluídos. Os estudos incluídos foram utilizados para a extração de informações/dados e seguiram para uma última fase, em que foi realizada uma análise qualitativa dos estudos. Além das bases descritas, artigos identificados nas listas de referências dos artigos selecionados ou de outras revisões e estudos relacionados ao tema passaram por esse processo de seleção.

\section{Extração dos dados}

Dos estudos incluídos foram extraídos como dados: autores do estudo; ano de publicação; número de participantes (n); faixa etária; diagnóstico de sobrepeso/obesidade; tipo de treinamento físico; duração do treinamento; frequência semanal das sessões de treino; duração das sessões; pressão arterial pré e pós-treinamento. A sumarização das informações coletadas foi organizada em tabela, e os dados da mesma foram todos apresentados conforme tinham sido disponibilizados nas publicações originais.

\section{Análise qualitativa dos estudos}

Para avaliação da qualidade metodológica dos ensaios clínicos randomizados controlados foi utilizada a Escala de qualidade PEDro. Essa escala consiste em 11 critérios relacionados à cegamento dos participantes e avaliadores, randomização do estudo e análise dos dados na linha de base, e assim, está diretamente relacionada com a redução dos riscos de viés. A pontuação final da escala de qualidade PEDro é dada por meio da soma do número de critérios que foram classificados como satisfatórios entre os critérios 2 ao 11. A pontuação final pode variar entre 0 e 10 pontos (Shiwa et al., 2011). Um estudo com uma pontuação PEDro de 7 foi considerado evidência de nível 1 (7-8 bom, 9-10 excelente) e um estudo com uma pontuação de 6 foi considerado evidência de nível 2 (5-6 aceitável, $\leq 4$ ruim) (Gambassi et al., 2019).

\section{Resultados}

\section{Estudos selecionados}

Um total de 621 estudos foi recuperado pela estratégia de busca usada nas bases de dados PUBMED (170 estudos); SCOPUS (165 estudos); WEB OF SCIENCE (126 estudos); COCHRANE LIBRARY (147 estudos); e LILACS (13 estudos). Além destes, mais 4 (quatro) estudos foram identificados nas listas de referência dos estudos recuperados, resultando em total de 625 estudos. Para verificação de duplicidade entre os estudos recuperados, os mesmos foram ex portados para o programa gerenciador de referências Mendeley ${ }^{\circledR}$, onde foram encontrados 196 estudos duplicados, restando assim um total de 429 registros após remoção de duplicidades. O processo de triagem e os critérios utilizados para elegibilidade dos estudos estão sumarizados e ilustrados no fluxograma abaixo (Figura 1). Depois de encerrado o processo de seleção, 8 (oito) estudos restantes foram incluídos na análise qualitativa e fizeram parte da presente revisão sistemática. 
Figura 1. Fluxograma elaborado pelo Preferred Reporting Items for Systematic Reviews and Metanalyses - PRISMA (Moher et al., 2009), demonstrando o processo de seleção dos estudos $(n=8)$ incluídos nesta revisão sistemática.

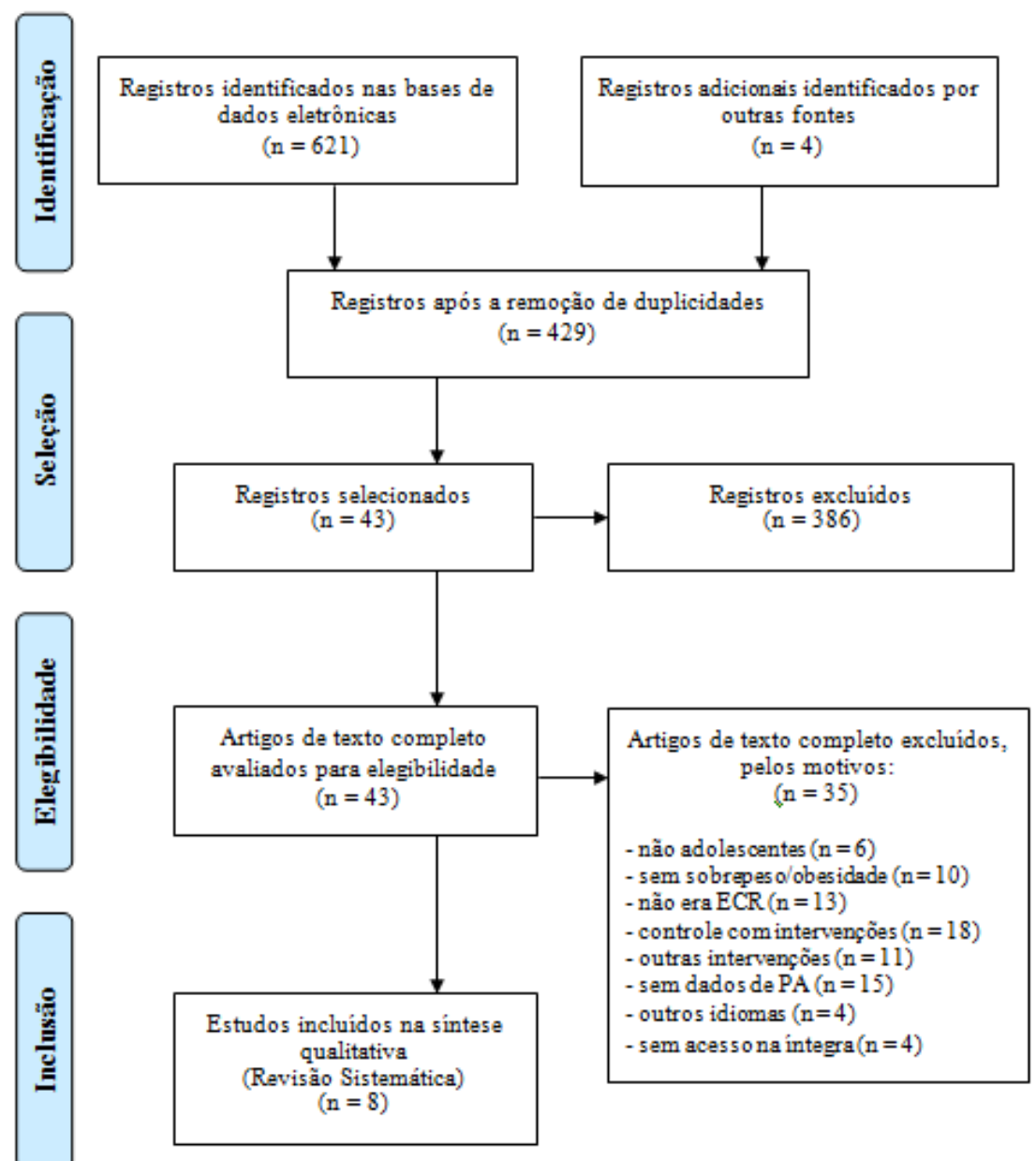

Fonte: Autores.

\section{Avaliação qualitativa dos estudos}

$\mathrm{Na}$ avaliação da qualidade metodológica a pontuação média na escala de qualidade PEDro foi de 5,6 com pontuação mínima de 4 e máxima de 7. Apenas o estudo de Cvetković et al (2018) foi considerado uma evidência de nível 1 - boa, os demais foram considerados evidência de nível 2. A alocação aleatória ocorreu em $75 \%$ dos estudos, pois dois estudos (Naylor et al., 2008; Lee et al., 2010) não descreveram o método de aleatorização dos grupos. Os estudos não relataram se houve alocação secreta, e todos dividiram os grupos em características semelhantes entre eles. Baixa qualidade dos estudos foi identificada em relação ao cegamento dos sujeitos, da equipe e dos resultados (12,5\%). Todos tiveram menos de $15 \%$ de perda amostral, 87,5\% descreveram diferença entre grupos na análise estatística e as medidas de tendência de variabilidade foram descritas em 100\%. A análise por intenção de tratar ocorreu em 75\% dos estudos.

\section{Características dos estudos}

Os estudos incluídos na síntese qualitativa do presente estudo apresentaram um total de 249 adolescentes obesos ou com sobrepeso, divididos em 156 do sexo masculino e 78 do feminino. Vale ressaltar que nessa divisão, o artigo de Jeon et al (2013) não relatou o sexo dos seus 15 participantes. Três trabalhos, Wong et al (2008); Kelly et al (2015) e Cvetković et al (2018) desenvolveram seus estudos apenas com adolescentes do sexo masculino, e apenas um Bharath et al (2018), trabalhou exclusivamente com voluntários do sexo feminino. Em relação ao estágio maturacional, apenas três estudos o avaliaram, Ounis 
et al (2010); Kelly et al (2015) e Bharath et al (2018), sendo que os três utilizaram os estágios maturacionais de Tanner para avaliação. Podemos descrever que nos estudos incluídos e que utilizaram essa avaliação, o estadiamento foi verificado entre 1 -4 .

Para classificar os voluntários em obesos e sobrepesos, todos os estudos incluídos utilizaram o IMC relativo ao sexo e idade, sendo que os estudos de Lee et al (2010); Jeon et al (2013) e Kelly et al (2015) descreveram em percentis os índices considerados como obeso ou sobrepeso, considerando os participantes entre o $85^{\circ}$ e $94^{\circ}$ percentil como sobrepesos e maiores ou iguais ao $95^{\circ}$ percentil como obesos. Os estudos de Ounis et al (2010) e Cvetković et al (2018) também consideraram os percentis relacionados ao sexo e idade dos participantes, porém esses percentis foram embasados por Cole et al (2000). O restante dos trabalhos considerou o valor bruto do IMC para classificação, sendo IMC $\geq 25$ e $<30$ considerados sobrepesos e IMC $\geq 30$ obesos.

Nenhum dos estudos selecionados tinha como objetivo primário o efeito do treinamento nos valores de pressão arterial, porém todos apresentaram esses valores como complementos do efeito do treinamento proposto na saúde cardiovascular de seus participantes. Três estudos, Lee et al (2010); Ounis et al (2010) e Cvetković et al (2018), se utilizaram do treinamento físico aeróbio como intervenção, dois estudos, Naylor et al (2008) e Kelly et al (2015), do treinamento físico de força ou de resistência à força (treinamento resistido) e quatro estudos, Wong et al(2008); Lee et al (2010); Jeon et al (2013) e Bharath et al (2018), da combinação dos treinamentos, aeróbio e de força, para responderem seus objetivos. Destes, cinco estudos, Naylor et al (2008); Wong et al (2008); Lee et al (2010); Ounis et al (2010) e Jeon et al (2013) apresentaram diferenças significativas em seus valores de pressão arterial após intervenção do treinamento quando comparados aos valores aferidos anteriormente a intervenção ou pela linha do tempo ao grupo controle (Quadro 2). 
Research, Society and Development, v. 10, n. 9, e4410917623, 2021

(CC BY 4.0) | ISSN 2525-3409 | DOI: http://dx.doi.org/10.33448/rsd-v10i9.17623

Quadro 2. Características gerais dos estudos avaliados sobre o efeito do treinamento físico na pressão arterial de adolescentes com excesso de peso.

\begin{tabular}{|c|c|c|c|c|c|c|}
\hline $\begin{array}{l}\text { Autor } \\
\text { (ano) }\end{array}$ & $\begin{array}{l}\text { Características } \\
\text { dos participantes }\end{array}$ & $\begin{array}{l}\text { Metodologia } \\
\text { do Estudo }\end{array}$ & $\begin{array}{l}\text { Duração } \\
\text { Semanas }\end{array}$ & $\begin{array}{l}\text { Tipo de } \\
\text { Exercício }\end{array}$ & \begin{tabular}{|c|}
$\begin{array}{c}\text { Instrumento e } \\
\text { protocolo de aferição } \\
\text { da PA }\end{array}$ \\
\end{tabular} & Resultados \\
\hline $\begin{array}{l}\text { Bharath } \\
\text { et al. } \\
\text { (2018) }\end{array}$ & $\begin{array}{c}40 \text { adolescentes } q \text { obesas } \\
\text { Controle: } \mathrm{n}=20(14,8 \pm 4,5 \text { anos }) \\
\text { Intervencão: } \mathrm{n}=20(14,6 \pm 4,5 \text { anos }) \\
\text { Estágio de Tanner: } 3-4\end{array}$ & $\begin{array}{c}5 \text { min de aquecimento } \times 20 \text { min de exercícios } \\
\text { contra resistência com elásticos ( } 40 \text { a } 70 \% \\
\text { FCreserva) x } 30 \text { min de caminhada e/ou } \\
\text { corrida em esteira } 60 \text { a } 70 \% \text { FCreserva x } 5 \\
\text { min de volta a calma }\end{array}$ & $\begin{array}{l}5 \mathrm{x} / \text { semana de } \\
60 \mathrm{~min} \text { por } 12 \\
\text { semanas }\end{array}$ & TC & Nāo relatado & 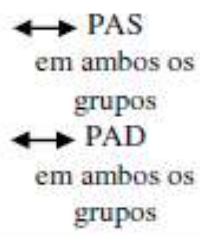 \\
\hline $\begin{array}{l}\text { Cvetković } \\
\text { et al. } \\
\text { (2018) }\end{array}$ & $\begin{array}{c}35 \text { adolescentes } \hat{\delta} \text { com excesso de peso } \\
\text { (11 a } 13 \text { anos) } \\
\text { Controle: } n=14 \\
\text { FUTEBOL: } n=10 \\
\text { HIIT: } n=11\end{array}$ & $\begin{array}{l}\text { FUTEBOL: } 10 \text { min de aquecimento } x 4 \text { jogos } \\
\text { de } 8 \text { min }(\sim 75 \% \text { FCmáx) intercalados com } \\
2 \text { min de descanso passivo } \times 10 \mathrm{~min} \text { de } \\
\text { relaxamento } \\
\text { HIIT: } 10 \mathrm{~min} \text { de aquecimento } \times 3 \text { séries de } 5- \\
10 \text { tiros de corrida por } 10 \text { a } 20 \text { seg com } \\
\text { mesmo intervalo de descanso com } 3 \mathrm{~min} \text { de } \\
\text { descanso passivo entre as séries }(\sim 80 \% \\
\text { FCmáx) } \times 10 \text { min de relax amento }\end{array}$ & $\begin{array}{c}\text { FUTEBOL: } \\
3 \mathrm{x} / \mathrm{semana} \text { de } \\
60 \mathrm{~min} \text { por } 12 \\
\text { semanas } \\
\text { HIIT: } 3 \mathrm{x} / \mathrm{semana} \\
\text { de } 35 \text { a } 50 \text { min por } \\
12 \text { semanas }\end{array}$ & TA & $\begin{array}{c}\text { Após } 5 \text { min de repouso } \\
\text { em posiçāo sentada, } \\
\text { utilizando } \\
\text { esfigmomanome tro } \\
\text { manual }\end{array}$ & 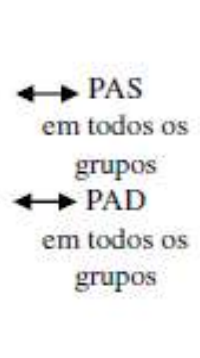 \\
\hline $\begin{array}{l}\text { Kelly } \\
\text { et al. } \\
(2015)\end{array}$ & $\begin{array}{c}26 \text { adolescentes } \delta \text { obesos } \\
\text { Controle: } n=13(15,6 \pm 0,9 \text { anos }) \\
\text { Intervencāo: } n=13(15,4 \pm 0,9 \text { anos }) \\
\text { Estágio de Tanner: } \geq 3\end{array}$ & $\begin{array}{c}1 \text { a } 4 \text { séries de } 8 \text { a } 15 \text { repetiçōes de leve a alta } \\
\text { intensidade realizadas na casa do voluntário } \\
\text { (home-based) }\end{array}$ & $\begin{array}{l}2 \mathrm{x} / \mathrm{semana} \text { de } \\
\sim 60 \mathrm{~min} \text { por } 16 \\
\text { semanas }\end{array}$ & TF & Nāo relatado & $\begin{array}{l}\leftrightarrow \text { PAS } \\
\text { em ambos os } \\
\text { grupos } \\
\leftrightarrow \text { PAD } \\
\text { em ambos os } \\
\text { grupos }\end{array}$ \\
\hline $\begin{array}{l}\text { Jeon } \\
\text { et al. } \\
(2013)\end{array}$ & $\begin{array}{l}15 \text { adolescentes com sobrepeso } \\
\text { Controle: } n=7 \\
\text { Intervencão: } n=8\end{array}$ & $\begin{array}{l}50 \text { min de exercícios contra resistência com } \\
\text { elásticos (70\% } 1 \text { RM) x } 30 \text { a } 35 \text { min de } \\
\text { caminhada e/ou corrida em esteira } 55 \text { a } 75 \% \\
\text { Fcmáx }\end{array}$ & $\begin{array}{l}2 \mathrm{x} / \mathrm{semana} \text { de } \\
290 \mathrm{~min} \text { por } 12 \\
\text { semanas }\end{array}$ & $\mathrm{TC}$ & $\begin{array}{c}\text { Após } 10 \mathrm{~min} \text { de } \\
\text { repouso em posiçāo } \\
\text { sentada, utilizando um } \\
\text { dispositivo automático } \\
\text { (DINMAP) com um } \\
\text { manguito de tamanho } \\
\text { adequado. }\end{array}$ & $\begin{array}{c}\downarrow \text { PAS } \\
\text { no grupo } \\
\text { Intevenção } \\
\leftrightarrow \text { PAD } \\
\stackrel{\text { em ambos os }}{\text { grupos }}\end{array}$ \\
\hline
\end{tabular}




\begin{tabular}{|c|c|c|c|c|c|c|}
\hline $\begin{array}{c}\text { Lee } \\
\text { et al. } \\
(2010)\end{array}$ & $\begin{array}{c}54 \text { adolescentes obesos }(q=9 ; \delta=45) \\
\text { (12 a } 14 \text { anos) } \\
\text { Controle: } n=18 \\
\text { AERÓBIO: } n=16 \\
\text { COMBINADO: } n=20\end{array}$ & $\begin{array}{c}\text { AERÓBIO: } 5 \text { min de aquecimento } x \\
\text { variedade de exercícios aeróbios como } \\
\text { futebol, basquete, futebol americano, } \\
\text { be isebol, hóquei, badminton, ginástica, pular } \\
\text { corda e alpinismo (70 a 90\% FCmáx) x } 5 \text { min } \\
\text { de relaxamento } \\
\text { COMBINADO: } 5 \text { min de aquecimento } x \\
\sim 15 \text { min de treinamento com pesos em } \\
\text { circuito } 2 \text { séries ( } 8 \text { a } 10 \text { estaçoes) } 30 \text { seg por } \\
\text { estaçāo com descanso de } 10 \text { seg entre cada } \\
\text { (70 a } 80 \% \text { da força máxima) } x \sim 35 \text { min de } \\
\text { exercícios aeróbios } x \text { min de relaxamento }\end{array}$ & $\begin{array}{l}\text { AERÓBIO: } \\
3 \mathrm{x} / \mathrm{semana} \text { de } \\
\sim 60 \mathrm{~min} \text { por } 10 \\
\text { semanas } \\
\text { COMBINADO: } \\
3 \mathrm{x} / \mathrm{semana} \text { de } \\
\sim 60 \mathrm{~min} \text { por } 10 \\
\text { semanas }\end{array}$ & TA e TC & $\begin{array}{l}\text { Após } 5 \text { min de repouso } \\
\text { em posição sentada, } \\
\text { utilizando monitor } \\
\text { oscilométrico. com o } \\
\text { manguito envolvendo } \\
\text { completamente a parte } \\
\text { superior do braço }\end{array}$ & $\begin{array}{l}\downarrow \text { PAS } \\
\text { em ambos os } \\
\text { grupos de } \\
\text { Intevenção }\end{array}$ \\
\hline $\begin{array}{l}\text { Ounis } \\
\text { et al. } \\
(\mathbf{2 0 1 0})\end{array}$ & $\begin{array}{c}32 \text { adolescentes obesos }(q=17 ; \delta=15) \\
\text { Controle: } n=16(13,2 \pm 0,6 \text { anos }) \\
\frac{\text { Intervença:o: } n=16(13,4 \pm 0,4 \text { anos })}{\text { Estágio de Tanner: } 1-4}\end{array}$ & $\begin{array}{c}\text { Aquecimento } x \text { brincadeiras diversas que } \\
\text { envolviam correr e saltar } x \text { relaxamento } \\
(\sim 90 \text { min). A intensidade foi fixada na FC } \\
\text { correspondente ao valor do FAT MAX } \\
\text { ( } 66 \% \text { VO2pico) }\end{array}$ & $\begin{array}{c}4 x / \text { semana de } \\
\sim 90 \text { min por } 8 \\
\text { semanas }\end{array}$ & TA & $\begin{array}{l}\text { Após } 5 \text { min de repouso } \\
\text { em posição sentada, } \\
\text { utilizando } \\
\text { esfigmomanômetro } \\
\text { aneróide, com } \\
\text { manguito de tamanho } \\
\text { apropriado }\end{array}$ & $\begin{array}{l}\downarrow \text { PAS } \\
\text { no grupo } \\
\text { Intevenção } \\
\downarrow \text { PAD } \\
\text { no grupo } \\
\text { Intervenção }\end{array}$ \\
\hline $\begin{array}{l}\text { Naylor } \\
\text { et al. } \\
(\mathbf{2 0 0 8})\end{array}$ & $\begin{array}{c}23 \text { adolescentes obesos }(q=12 ; \delta=11) \\
\text { Controle: } \mathrm{n}=10(13,6 \pm 2,2 \text { anos }) \\
\underline{\text { Intervenção: } \mathrm{n}=13(12,2 \pm 1,4 \text { anos })}\end{array}$ & $\begin{array}{c}\text { 10min de aque cimento } x \sim 40 \mathrm{~min} \text { de circuito } \\
\text { de treinamento de força em máquinas de } \\
\text { peso } 2 \text { séries em } 10 \text { estaçóes } 1 \text { min por } \\
\text { estação ( } 8 \text { rep) com descanso de } 1 \text { min entre } \\
\text { cada ( } 75 \text { a } 90 \% \text { da força máxima) x } 10 \mathrm{~min} \\
\text { de relaxamento }\end{array}$ & $\begin{array}{c}3 \mathrm{x} / \mathrm{semana} \text { de } \\
60 \mathrm{~min} \text { por } 8 \\
\text { semanas }\end{array}$ & TF & Nào relatado & $\begin{array}{c}\downarrow \text { PAS } \\
\text { no grupo } \\
\text { Intevenção } \\
\longleftrightarrow \text { PAD } \\
\text { em ambos os } \\
\text { grupos }\end{array}$ \\
\hline $\begin{array}{l}\text { Wong } \\
\text { et al. } \\
\text { (2008) }\end{array}$ & $\begin{array}{c}24 \text { adolescentes } \delta \text { obesos } \\
\text { Controle: } n=12(14,3 \pm 1,5 \text { anos }) \\
\text { Intervença: } n=12(13,8 \pm 1,1 \text { anos })\end{array}$ & $\begin{array}{c}\text { } 5 \text { min de aquecimento } \times 45 \text { a } 60 \mathrm{~min} \text { de uma } \\
\text { variedade de atividades compreendendo uma } \\
\text { combinação de exercícios aeróbicos e contra } \\
\text { resistência em circuito, e atividades de jogo } \\
\text { como futebol, handebol, exercícios de subir } \\
\text { escadas e outras atividades recreativas ativas } \\
\text { envolvendo sessós de trabalho contínuo }(65 \\
\text { a } 85 \% \text { de FCmax x } 5 \text { min de relaxamento }\end{array}$ & $\begin{array}{l}2 x / \text { semana de } \\
\sim 60 \text { min por } 12 \\
\text { semanas }\end{array}$ & TC & Nao relatado &  \\
\hline
\end{tabular}

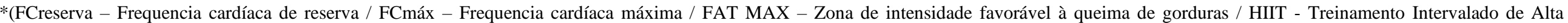


Autores 


\section{Discussão}

O objetivo desta revisão foi analisar os efeitos do treinamento físico na pressão arterial de adolescentes com sobrepeso e obesidade. Nossos principais resultados sugerem que o treinamento físico é capaz de reduzir os valores de pressão arterial, independentemente do tipo, sendo ele de características aeróbias, de força, ou a combinação das duas. Os estudos apontam para uma maior redução da PA induzida após treinamento aeróbio quando comparado ao treinamento combinado e de força. Em comparação com o treinamento de força, o treinamento combinado levou a uma queda mais acentuada da resposta hipotensiva.

O efeito do treinamento aeróbio foi investigado por Lee et al (2010); Ounis et al (2010) e Cvetković et al (2018). Lee et al (2010) avaliaram adolescentes obesos de ambos os sexos após treinamento aeróbio (futebol, basquete, futebol americano, beisebol, hóquei, badminton, ginástica, pular corda e alpinismo) a 70 - 90\% da FCmáx por aproximadamente 60min, 3 vezes por semana por 10 semanas, e encontraram redução de mais $7 \mathrm{mmHg}$ na PAS. Ounis et al (2010) com público bem parecido ao estudo anterior, também encontraram redução significativa de mais de $10 \mathrm{mmHg}$ na PAS e quase $9 \mathrm{mmHg}$ na PAD após treinamento aeróbio (brincadeiras diversas que envolviam correr e saltar) na intensidade do FATMAX por aproximadamente 90min, 4 vezes por semana por 8 semanas.

Porém, Cvetković et al (2018) analisando apenas adolescentes do sexo masculino, não encontraram reduções significativas na PA após treinamento aeróbio (4 partidas de futebol de 8min cada) a aproximadamente 75\% da FCmáx, 3 vezes por semana por 12 semanas. Os protocolos de treinamento realizados nos estudos incluídos variam amplamente em volume prescritos e, portanto, a literatura ainda carece de evidências para afirmar se o volume e a intensidade influenciam na magnitude das adaptações crônicas do exercício físico na pressão arterial de adolescentes com excesso de peso.

Os efeitos crônicos nos valores de pressão arterial resultam da adaptação do organismo à exposição frequente e regular ao exercício físico proposto, sendo vários os mecanismos que explicam esse efeito hipotensor e protetor do exercício físico, praticado de forma regular, na saúde cardiovascular, dentre eles as adaptações neuro-hormonais que ocorrem nos vasos sanguíneos, músculos e adipócitos, como, a redução dos níveis de noradrenalina circulante e seus receptores; aumento da biodisponibilidade de óxido nítrico; aumento da capacidade antioxidante; aumento da sensibilidade à insulina; redução da frequência cardíaca de repouso, entre outras. Além de adaptações estruturais como, a remodelagem vascular (aumento do comprimento, lúmen vascular e número de esfíncteres pré-capilares); angiogênese; alterações anatômicas e funcionais do coração, entre outras (Pithon-Curi, 2013).

As reduções na PAS por meio do treinamento físico aeróbio estão bem estabelecidas na literatura, com reduções aproximadas de 6 a $10 \mathrm{mmHg}$ observadas em homens e mulheres previamente sedentários de todas as idades (Balas-Nakash et al., 2010; Farah et al., 2014; Bruñó et al., 2018). Um dos mecanismos fisiológicos primários que explicam a redução da PA após treinamentos de características predominantemente aeróbias é o que leva em consideração o padrão de recrutamento de grandes massas musculares, gerando um acentuado aumento no consumo de oxigênio e débito cardíaco, mediante ao aumento do volume diastólico final, bem como o aumento do estado contrátil do músculo cardíaco, reduzindo assim o volume sistólico final. Temos que, a pressão arterial sistólica se eleva, ao passo que a diastólica e média permanecem em constância, reduzindo assim a resistência vascular periférica, sobrecarga essa com predominância de volume, que propicia aumento da parede ventricular esquerda com proporcional aumento no diâmetro cavitário, induzindo hipertrofia fisiológica excêntrica do ventrículo esquerdo, e posterior redução da PAS em repouso (Ghorayeb et al., 2005).

O efeito do treinamento de força foi investigado pelos estudos de Naylor et al (2008) e Kelly et al (2015). Os primeiros avaliaram adolescentes obesos de ambos os sexos após treinamento de força em circuito (2 séries de 8 repetições em 10 estações de exercícios) a 75 - 90\% de 1RM, por uma hora, 3 vezes por semana, durante 8 semanas, e econtraram redução significativa de aproximadamente $4 \mathrm{mmHg}$ na PAS. Entretanto, o estudo de Kelly et al (2015) não econtrou reduções siginificativas na PA após 16 semanas de treinamento de força (home-based) em adolescentes do sexo masculino, 60min, 2 
vezes por semana, de 1 a 4 séries de 8 a 15 repetições de leve a alta intensidade (intensidade progressiva) orientado e realizado na casa do voluntário. Mais uma vez cabe destacarmos os diferentes volumes e intensidades dos estudos, o que provavelmente influencia os resutados distintos encontrados entre eles.

Os mecanismos pelos quais o treinamento de força pode induzir adaptações favoráveis na PA na juventude são especulativos. Alguns estudos especularam que a redução da PA após o TF em jovens pode ser devido ao aumento da massa muscular esquelética que, por sua vez, pode levar a um relaxamento miocárdico (Naylor et al., 2008), uma melhora na modulação autonômica (Farinatti et al., 2016) e/ou uma função endotelial aprimorada (Yu et al., 2016). Não obstante, Ghorayeb et al (2005), coloca que os exercícios que demandam força contra uma resistência externa, comumente utilizam-se de massas musculares menores quando equiparados com os exercícios aeróbios e, portanto, causam um aumento inferior do débito cardíaco e menor consumo de oxigênio, com reduzido aumento do volume sistólico, porém, com acentuada elevação das pressões arteriais, tanto a sistólica como a diastólica e média, com aumento da resistência vascular periférica. Essa sobrecarga de trabalho apresenta características de pressão que geram espessamento da parede ventricular esquerda, desencadeando hipertrofia fisiológica concêntrica do ventrículo esquerdo, podendo gerar, ou não, uma possível redução da PAS em repouso.

O efeito da combinação de ambos os treinamentos (aeróbio + força) foi investigado por Wong et al (2008); Lee et al (2010); Jeon et al (2013) e Bharath et al (2018). Wong et al (2008) avaliaram adolescentes obesos do sexo masculino após 12 semanas de treinamento combinado [treinamento de força em circuito + exercícios aeróbios (futebol; handebol; subir e descer escadas...)] de 45 a 60min, 2 vezes por semana, em intensidade progressiva de 65-85\% da FCmáx, e encontraram redução significativa de quase $6 \mathrm{mmHg}$ na PAS. Não obstante, Lee et al (2010) avaliaram adolescentes obesos de ambos os sexos após treinamento combinado [(treinamento com pesos em circuito (2 séries em 8-10 estações por 30seg em cada estação) + exercícios aeróbios)] aproximadamente 60min, 3 vezes por semana por 10 semanas, e também encontraram redução de mais de $9 \mathrm{mmHg}$ na PAS. Entretanto, Bharath et al (2018) após 12 semanas de treinamento combinado [(exercícios contra-resitência com elásticos (40-70\% FCreserva) + caminhada ou corrida (60-70\% FCreserva)] com adolescentes do sexo feminino, 60min por sessão, 5 sessões por semana, não encontraram reduções significativas na PA após treinamento.

Em se tratando de adolescentes com excesso de peso e as complicações cardiovasculares e metabólicas ocasionadas pelo excesso de adiposidade, como, pressão alta, colesterol alto e síndrome metabólica, devemos considerar que a estratégia dos programas de treinamento físico, buscada em primeiro lugar, é para redução do peso, em especial de massa de gordura. E para isso, o treinamento combinado tem se mostrado bastante eficiente melhorando a força geral, variáveis metabólicas e a aptidão cardiorrespiratória e reduzindo percentual de gordura corporal, circunferência da cintura, resistência à insulina, rigidez arterial e pressão arterial em diferentes idades (Antunes et al., 2015; Son et al., 2017; Bharath et al., 2018).

A presente revisão apresenta algumas limitações relacionadas ao período do treinamento físico ser concomitante às aulas de educação física. Alguns autores relataram a participação de seus voluntários nas aulas e outros não descreveram se os participantes estavam apenas em período de treinamento físico proposto ou simultâneo às aulas de educação física. Em se tratando de adolescentes em idade escolar, pressupomos a participação nas aulas a todos os participantes. Outra limitação do estudo foi a não possibilidade de geração da metanálise e consequentemente significância estatisticamente comprovada aos dados e comparações objetivadas no presente estudo.

Além disso, os estudos incluídos na avaliação da qualidade metodológica, apresentaram uma pontuação média na escala de qualidade PEDro de 5,6, sendo que apenas 1 estudo (Cvetković et al., 2018) foi considerado uma evidência de nível 1 - boa, os demais foram considerados evidência de nível 2 - aceitável ou ruim (Gambassi et al., 2019). A qualidade metodológica pode comprometer os achados do presente estudo, uma vez que a média do escore PEDro se encontra na classificação de estudos "aceitáveis" o que não confere uma boa qualidade. 
Apesar do exposto, esta é a primeira revisão sistemática que temos conhecimento a propor identificar quais tipos de treinamento físico têm mais efetividade na redução dos níveis pressóricos de adolescentes com excesso de peso. Um ponto forte é que o escopo desta revisão foi limitado a estudos que não incluíram intervenções dietéticas, medicamentosas, entre outras, o que nos possibilitou analisar o efeito apenas do treinamento físico sobre a PA. Outro ponto forte é não inclusão de grupos controles com peso normal, enfatizando os efeitos do treinamento físico em adolescentes com sobrepeso e obesidade. Estudos futuros que enfocam a redução da PA após intervenção com treinamento físico devem usar estratégias que podem ajudar a garantir a comparabilidade entre os estudos e maximizar o potencial de harmonização de dados. Estudos multiníveis que examinam os efeitos do tipo de treinamento físico na redução da PA de adolescentes com excesso de peso também são recomendados para explicar as possíveis diferenças observadas entre os programas.

\section{Conclusão}

Conclui-se que o treinamento físico, independentemente do tipo, é eficaz em reduzir os valores de pressão arterial, principalmente PAS, em adolescentes com sobrepeso e obesidade, principalmente quando este é realizado entre 8 e 16 semanas, de 2 a 5 vezes por semana, 30 a 90 minutos por dia em intensidade para exercícios aeróbios de 50 a $90 \%$ da FCmáx e para exercícios de força de 70 a 90\% de 1RM. Assim o treinamento físico pode ser uma estratégia eficaz no controle da hipertensão arterial em adolescentes com excesso de peso. Embora os valores de pressão arterial tendam a apresentar maior redução quando realizado o treinamento aeróbio em comparação ao treinamento de força ou combinado, a literatura carece de consenso sobre as características de cada tipo de treinamento e a melhor estratégia de prescrição.

Trabalhos futuros que enfatizem a redução da pressão arterial em adolescentes com excesso de peso após treinamento físico de maior durabilidade (> 16 semanas) e o efeito do "des"treinamento nos valores pressóricos, além de programas de treino metodologicamente claros quanto às suas características fisiológicas objetivadas, poderão contribuir na elucidação de um consenso clinico/prático para os profissionais que atuam com esse público.

\section{Referências}

Antunes, B. M. M., et al. (2015). Effect of concurrent training on gender-specific biochemical variables and adiposity in obese adolescents. Archives of endocrinology and metabolism, 59(4), 303-309.

Balas-Nakash, M., et al. (2010). The effect of exercise on cardiovascular risk markers in Mexican school-aged children: comparison between two structured group routines. Salud publica de Mexico, 52(5),398-405.

Bharath, L. P., et al. (2018). Combined resistance and aerobic exercise training reduces insulin resistance and central adiposity in adolescent girls who are obese: randomized clinical trial. European journal of applied physiology, 118(8), 1653-1660.

BRASIL (2012). Diretrizes Metodológicas: elaboração de revisão sistemática e metanálise de ensaios clínicos randomizados. (1a Ed). Brasília: Ministério da Saúde.

Bruñó, A., et al. (2018). Home-exercise Childhood Obesity Intervention: A Randomized Clinical Trial Comparing Print Versus Web-based (Move It) Platforms. Journal of pediatric nursing, 42, e79-e84.

Cole, T. J., et al. (2000). Establishing a standard definition for child overweight and Obesity Worldwide : International Survey. BMJ,320, (table 1), 1-6.

Cvetković, N., et al. (2018). Exercise training in overweight and obese children: Recreational football and high-intensity interval training provide similar benefits to physical fitness. Scandinavian journal of medicine \& science in sports, 28 (Suppl 1), 18-32.

Dias, I., et al. (2015). Effects of Resistance Training on Obese Adolescents. Medicine and science in sports and exercise, 47(12), 2636-2644.

Farah, B. Q., et al. (2012). Effect of physical training on the blood pressure of adolescents with obesity. Revista Paulista de Pediatria, 30(4), $600-607$.

Farah, B. Q., et al. (2014). Does exercise intensity affect blood pressure and heart rate in obese adolescents? A 6-month multidisciplinary randomized intervention study. Pediatric obesity, 9(2), 111-120.

Farinatti, P., et al. (2016). Short-term resistance training attenuates cardiac autonomic dysfunction in obese adolescents. Pediatric Exercise Science, 28(3), 374-380. 
Gambassi, B. B., et al. (2019). Effects of Exercise Training on Cardiovascular and Autonomic Parameters in Stroke Survivors: A Systematic Review. International Journal of Cardiovascular Sciences, 32(4), 408-413.

García-Hermoso, A., et al. (2013). Effects of exercise on resting blood pressure in obese children: A meta-analysis of randomized controlled trials. Obesity Reviews, 14(11), 919-928.

García-Hermoso, A., et al. (2015). Effects of aerobic plus resistance exercise on body composition related variables in pediatric obesity: A systematic review and meta-analysis of randomized controlled trials. Pediatric Exercise Science, 27(4), 431-440.

García-Hermoso, A., et al. (2020). Association between Exercise-Induced Changes in Cardiorespiratory Fitness and Adiposity among Overweight and Obese Youth: A Meta-Analysis and Meta-Regression Analysis. Children, 7(9), 147.

Ghorayeb, N., et al. (2005). Left Ventricular Hypertrophy of Athletes. Adaptative Physiologic Response of the Heart. Arq Bra Cardiol,85(3), $191-197$.

Hutton, B., et al. (2015). The PRISMA extension statement for reporting of systematic reviews incorporating network meta-analyses of health care interventions: Checklist and explanations. Annals of Internal Medicine, 162(11), 777-784.

Jeon, J. Y., et al. (2013). The combined effects of physical exercise training and detraining on adiponectin in overweight and obese children. Integrative medicine research, 2(4), 145-150.

Kelly, L. A., et al. (2015). The effect of a home-based strength training program on type 2 diabetes risk in obese Latino boys. Journal of pediatric endocrinology \& metabolism, 28(3-4), 315-322.

Lee, S. J., et al. (2019). What Is the Role of Resistance Exercise in Improving the Cardiometabolic Health of Adolescents with Obesity? Journal of Obesity \& Metabolic Syndrome, 28(2), 76-91.

Lee, Y. H., et al. (2010). The effects of an exercise program on anthropometric, metabolic, and cardiovascular parameters in obese children. Korean circulation journal, 40(4), 179-184.

Liberati, A., et al. (2009). The PRISMA statement for reporting systematic reviews and meta-analyses of studies that evaluate health care interventions: explanation and elaboration. Journal of Clinical Epidemiology, 62, e1-e34.

Milano-Gai, G. E., et al. (2018). 12-Week aerobic exercise and nutritional program minimized the presence of the 64Arg allele on insulin resistance. Journal of pediatric endocrinology \& metabolism, 31(9), 1033-1042.

Moher, D., et al. (2009). Preferred reporting items for systematic reviews and meta-analyses: The PRISMA statement. BMJ (Online), 339(7716), 332-336.

Monteiro, P. A., et al. (2015). Concurrent and aerobic exercise training promote similar benefits in body composition and metabolic profiles in obese adolescents. Lipids in Health and Disease, 14(1), 1-9.

Naylor, L. H., et al. (2008). Resistance training and diastolic myocardial tissue velocities in obese children. Medicine and Science in Sports and Exercise, 40(12), 2027-2032.

Ounis, O. B., et al. (2010). Exercise improves the ApoB/ApoA-I ratio, a marker of the metabolic syndrome in obese children. Acta Paediatrica, International Journal of Paediatrics, 99(11), 1679-1685.

Pithon-Curi, T. C., (2013). Fisiologia do Exercício. Guanabara Koogan.

SBP (2019). Obesidade na Infância e Adolescência: Manual de Orientação. (3a Ed). Sociedade Brasileira de Pediatria.

Shiwa, S. R., et al. (2011). PEDro: a base de dados de evidências em fisioterapia. Fisioter. Mov., 24(3), 523-533.

Sigal, R. J., et al. (2014). Effects of aerobic training, resistance training, or both on percentage body fat and cardiometabolic risk markers in obese adolescents: The healthy eating aerobic and resistance training in youth randomized clinical trial. JAMA Pediatrics, 168(11), 1006-1014.

Son, W. M., et al. (2017). Combined exercise training reduces blood pressure, arterial stiffness, and insulin resistance in obese prehypertensive adolescent girls. Clinical and experimental hypertension, 39(6), 546-552.

Vasconcellos, F., et al. (2014). Physical activity in overweight and obese adolescents: Systematic review of the effects on physical fitness components and cardiovascular risk factors. Sports Medicine, 44(8), 1139-1152.

WHO - World Health Organization (2017). Global Accelerated Action for the Health of Adolescents (AA-HA!): guidance to support country implementation Summary. Geneva: World Health Organization.

WHO - World Health Organization (2018). Taking action on childhood obesity report. World Health Organization, 1-8.

WHO - World Health Organization (2020). Obesity and overweight. https://www.who.int/news-room/fact-sheets/detail/obesity-and-overweight.

Wong, P. C. H., et al. (2008). Effects of a 12-week exercise training programme on aerobic fitness, body composition, blood lipids and C-reactive protein in adolescents with obesity. Annals of the Academy of Medicine Singapore, 37(4), 286-293.

Yu, C. C. W., et al. (2016). Effects of resistance training on cardiovascular health in non-obese active adolescents. World J Clin Pediatr, 5(3), 293-300. 